\title{
Heterogeneous Integration of Flexible Substrate Spiral Antennas and Silicon Chips by Magnetic Coupling
}

\author{
Luca Aluigi, ${ }^{\#, *}$, Trang T. Thai ${ }^{*}$, Manos M. Tentzeris", Luca Roselli" ${ }^{\#}$ and Federico Alimenti\# \\ ${ }^{*}$ DIEI, University of Perugia, Via Duranti 93, 06125 Perugia, Italy \\ *GEDEC, Georgia Institute of Technology, Atlanta, 30332-0250, USA
}

\begin{abstract}
A new contactless assembly method for SoC wireless transceivers and antennas on flexible and paper substrates is presented. An Archimedean spiral antenna, matched to a heterogeneous transformer which couples the received power to the chip wirelessly has been simulated at $1.5 \mathrm{GHz}$. Finally, a proof-of-concept prototype was fabricated and experimented. The measurements of the complete structure including the antenna and the transformer show $\left|S_{11}\right|$ values of about $12 \mathrm{~dB}$ at $1.5 \mathrm{GHz}$.
\end{abstract}

Index Terms - Electromagnetic coupling, flexible electronics, heterogeneous integration, ink-jet printing, three-dimensional (3-D) integration.

\section{INTRODUCTION}

Radio frequency transceivers of future wireless products will be able to manage multi-standard (multi-band, multidata-rate, etc.) operation with high modularity, high integration, low power consumption and low fabrication costs [1]. A high integration level is the key to reduce both the PCB area and the Bill-of-Material (BoM) and then to produce a miniaturized transceiver that can be sold at prices even lower than the current ones. Furthermore, the multi-standard operation require a frequency agile front-end, i.e. a device that is able to cover all services in the range from $300 \mathrm{MHz}$ to $5.5 \mathrm{GHz}$ by avoiding circuit duplicates. To improve the state-of-the-art, a system with more than 10 frequency bands is required. The related circuitry should be mounted on a PCB with an area less than 200 square millimeters and using less than 70 components in the RF modem. In this perspective, one particular issue is constituted by the antenna. The antenna, indeed, is one of the largest building blocks in term of occupied area. This is because its size is constrained to be within a quarter to a half of wavelength for an acceptable radiation efficiency. From this observation one can simply infer that antennas useful for the above frequency range cannot be integrated directly on silicon, but must be implemented on a different substrate, like the modem PCB or a flexible (conformal) PCB.

The heterogeneous integration between an antenna and a System-on-Chip (SoC) is a problem that must be studied with care. Existing solutions, like the Chip-on-Board (CoB) approach reported in [2], are characterized by interconnection parasitics (mostly of inductive nature) that can affect the circuit performances at high frequencies. In addition a precise positioning of the chip with respect to the PCB is required to establish the galvanic contacts.

In this paper the alternative solution illustrated in [3] is adopted. With such an approach the chip is magnetically coupled to the antenna realized on the PCB substrate, thus eliminating all the galvanic contacts between the chip and the antenna itself. The magnetic coupling is established by a heterogeneous planar transformer, the primary and secondary windings of which are implemented on PCB substrate and Si chip, respectively. As a result, the chip can be mounted by merely placing and gluing processes. The base-band signal lines and the required bias lines can be provided by standard wire bonding without caring for parasitic inductances.

In particular, the proposed case-of-study focuses on the design of a planar spiral antenna that is magnetically coupled to the feed system. This antenna has been selected since its wide-band capability is well known [4]. The central operation frequency has been chosen around $1.5 \mathrm{GHz}$, thus very close to the GNSS services. A 250nm CMOS technology has been selected to discuss the secondary coil of the heterogeneous transformer that, with the adopted method, is implemented on chip. Finally, to explore ultra-low cost solutions, the antenna is fabricated on a paper substrate exploiting the inkjet printing method [5].

\section{SYSTEM DESCRIPTION}

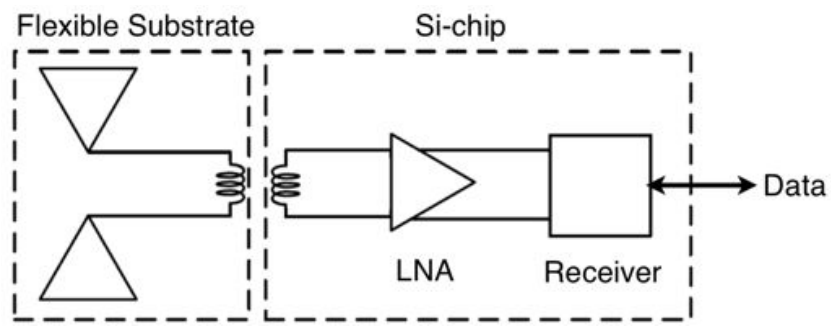

Fig. 1. Case-of-study: SoC wireless receiver magnetically-coupled to an antenna.

The case-of-study is a wireless receiver as illustrated in Fig. 1. The essential principle of the operation of the system is the magnetic coupling between the antenna and the chip. Such a contactless coupling is obtained by a heterogeneous transformer, the primary and secondary windings of which 
are fabricated on the antenna flexible substrate and Si chip respectively. On the Si-chip (i.e. on the receiver chip) the RF pads are substituted by the secondary coil of the heterogeneous transformer. This is realized by a planar winding connected to the receiver input circuitry. The DC supply and the data-out are then passed through the side of the substrate using wire bonds.

In our case-of-study the system operates around $1.5 \mathrm{GHz}$, a frequency close to GNSS navigation application services.

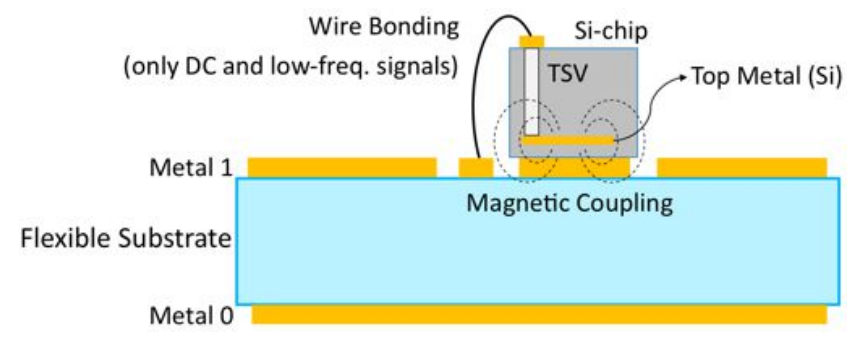

Fig. 2. Flex antenna to SoC transceiver assembly The heterogeneous transformer for magnetic coupling is formed between a coil on Top Metal (Si) and another coil on Metal 0 or Metal 1. The wire bondings are only for DC or low frequency signals.

Multiple layers of thin-film material can be arranged and laminated to form a package stackup similar to the one shown in Fig. 2. Metal 1 will be used for the antenna, the matching network and the primary coil. The top on-chip metal will be used for the secondary coil. Metal 0 will be used for the ground plane of the antenna (if needed) and/or as a further option for the primary coil.

\section{EM SiMULATIONS RESULTS}

In this section, the proposed idea is validated by means of full-wave EM simulations.

\section{A. Coupling Transformers}

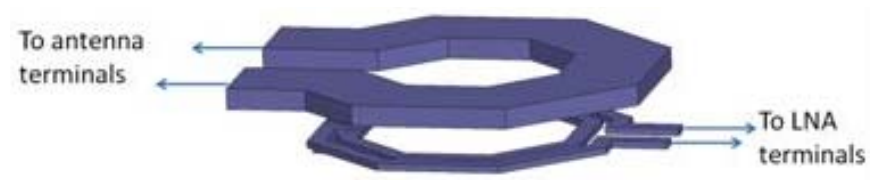

Fig. 3. Heterogeneous transformer. The upper coil (primary) is built on Metal 1 (see Fig. 2) and its terminals are connected to the antenna. The lower coil (secondary) is built on chip using the topmost metal available in the CMOS process.

First, a heterogeneous transformer between the flexible substrate and CMOS $\mathrm{Si}$ chip has been designed and simulated. The geometry of the transformer has been defined on the basis of the typical resolution capabilities of the flexible substrate fabrication facilities, which impose the lower limit on primary coil diameter and thus on the whole transformer dimensions. The two coils are separated by a distance of $25 \mu \mathrm{m}$ and have been placed face-to-face. This distance models the glue layer. For the secondary coil, a 250nm SiGe BiCMOS process has been taken into account, using the available thick top metal layers, named M5 and M4, respectively for the coil and underpass design. The two coils are shown in Fig. 3. They have an octagonal shape and the dimensions are summarized in Tab. I. The obtained maximum available power gain (MAG) of the transformer is $-0.52 \mathrm{~dB}$ at $1.5 \mathrm{GHz}$, thus indicating that, with the right matching, only $11 \%$ of the incident power is lost inside the transformer.

TABLE I

DIMENSIONS OF THE HETEROGENEOUS TRANSFORMER

\begin{tabular}{|c|c|c|c|c|c|}
\hline & $\begin{array}{c}\mathrm{D} \\
{[\mathrm{mm}]}\end{array}$ & $\begin{array}{c}\text { Width } \\
{[\mathrm{mm}]}\end{array}$ & $\begin{array}{c}\text { Spacing } \\
{[\mathrm{mm}]}\end{array}$ & $\begin{array}{c}\mathrm{N} \\
\text { turns }\end{array}$ & $\begin{array}{c}\text { Thick. } \\
{[\mu \mathrm{m}]}\end{array}$ \\
\hline Primary & 5 & 1 & - & 1 & 9 \\
\hline Secondary & 4.6 & 0.3 & 0.1 & 2 & 3 \\
\hline
\end{tabular}

\section{B. Antenna matched to the homogeneous transformer}

The two-wire Archimedean spiral configuration has been chosen for the antenna design due to its balance feed terminal and circular polarization. Additionally, the Archimedean configuration with the reflector allows the spiral antennas to achieve high gain with radiation pattern of a broadside antenna.

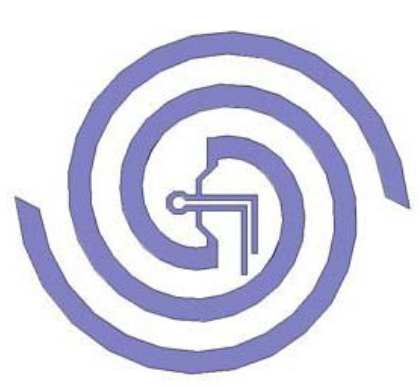

(a)

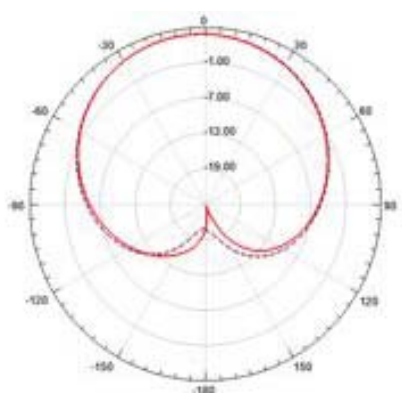

(b)
Fig. 4. (a) Top view of the designed antenna (lying on the $x-y$ plane) and (b) its radiation pattern. E-plane (x-z plane) in solid line, Hplane (y-z plane) in dashed line.

Fig. 4(a) shows the top view of the designed antenna. The secondary coil of the transformer, buried below the visible layer in figure (as shown in Fig. 3), couples the RF signal received by the antenna to the chip. The antenna reflector is made of the Metal 0 layer shown in Fig. 2. A feed network based on microstrip line is designed for the spiral antenna to allow maximum power transfer from the antenna to the coils and ultimately the chip. This network is constituted by the high-impedance connections and by the two open stubs (balanced structure) shown again in the layout of Fig. 4(a). The simulation port has been placed at the terminals of the secondary coil. The total gain of the structure from simulations is about $5 \mathrm{~dB}$ at $1.5 \mathrm{GHz}$. The radiation pattern is shown in Fig. 4(b) for both $\mathrm{E}$ - and $\mathrm{H}$ - planes. Tab. II summarizes the antenna dimensions. 
TABLE II

DIMENSIONS OF THE SPIRAL ANTENNA

\begin{tabular}{|c|c|c|c|c|}
\hline $\begin{array}{c}\mathrm{D} \\
{[\mathrm{cm}]}\end{array}$ & $\begin{array}{c}\text { Width } \\
{[\mathrm{mm}]}\end{array}$ & $\begin{array}{c}\text { Spacing } \\
{[\mathrm{mm}]}\end{array}$ & $\begin{array}{c}\text { N turns } \\
(\text { each arm })\end{array}$ & $\begin{array}{c}\text { Thick. } \\
{[\mu \mathrm{m}]}\end{array}$ \\
\hline 8 & 5 & 5.5 & 1.25 & 9 \\
\hline
\end{tabular}

\section{EXPERIMENTAL RESULTS}

As a proof-of-concept to illustrate the effective contactless coupling of the coils and the power collecting capability at a given frequency band of the proposed design, a prototype was fabricated by inkjet printing on paper substrates and measured.

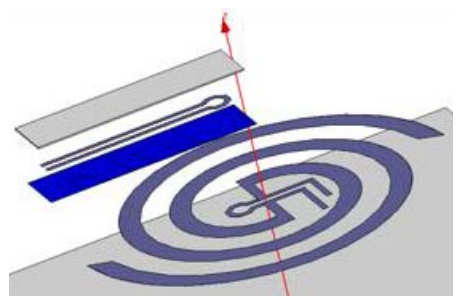

(a)

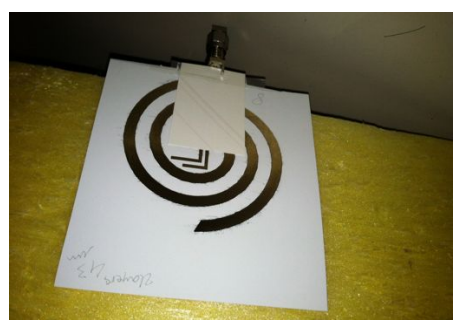

(b)

Fig. 5. (a) exploded view of the fabricated prototype. (b) capture of the fabricated prototype at $1.5 \mathrm{GHz}$ range.

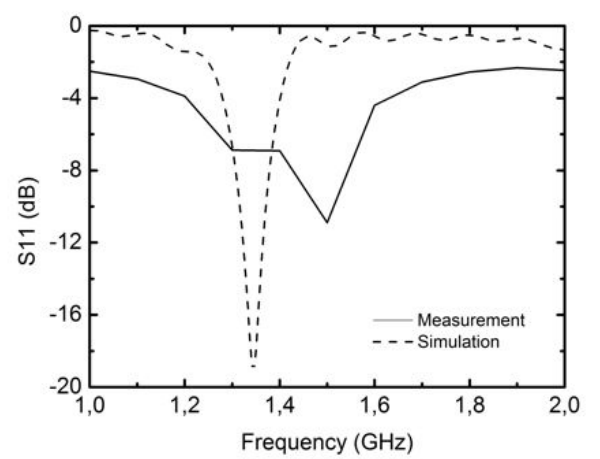

Fig. 6. Measured (solid-line) and simulated (dashed-line) $\mathrm{S}_{11}$ of the prototype antenna shown in Fig. 5.

Fig. 5(a) shows an exploded view of the measured prototype, shown in Fig. 5(b). The top layer visible in Fig. 5 (a) is a paper superstrate with thickness 220 um below which is built the secondary (1-turn) coil including the input lines. Below is the glue layer (in blue). A super glue layer having dielectric constant of 3.3 and loss tangent of 0.06 with the estimated thickness of $100 \mu \mathrm{m}$ was utilized to attach the superstrate to the substrate on which the antenna and the primary coil are built. The final assembled prototype is shown in Fig. 5(b). In this proof-of-concept the magnetic coupling has been implemented by a homogeneous transformer having both primary and secondary coil on flexible substrate. The two coils have the same dimensions as the primary coil in Tab. I. The simulated maximum available power gain (MAG) of the transformer is $-0.1 \mathrm{~dB}$ at $1.5 \mathrm{GHz}$, thus indicating that, with the right matching, only $3 \%$ of the incident power is lost inside the transformer.

The measurements of the prototype is shown in Fig. 6 giving the $|\mathrm{S} 11|$ of $-12 \mathrm{~dB}$ at $1.5 \mathrm{GHz}$. The discrepancy in the magnitude of the results between the simulated and measured data is due to mechanical manufacturing tolerances in the alignment of the two substrates, while the slight shift in the peak measurement is due to the instrument uncertainty in the glue thickness estimation.

\section{CONCLUSION}

In this paper a contactless assembly method for SoC wireless transceivers (Si-chip) and antennas on flexible or paper substrates is introduced and validated with EM simulations. Then a proof-of-the-concept structure is developed exploiting a spiral antenna on paper substrate. Such a structure is fabricated by conductive (silver) ink and ink-jet printing technology. The coupling transformer is also fabricated in paper and then glued on top of the antenna. The experimental results show the feasibility of the idea, thus enabling for a significant reduction in the manufacturing cost of future wireless products.

\section{ACKNOWLEDGEMENTS}

This work was partially supported by the "ARTEMOS" project (ENIAC-JU, call 32010 ), under the activity of Task 3.6. The research has been implemented under a Fulbright scholarship, Jun. - Dec. 2012, held at GaTech, Atlanta, USA.

\section{REFERENCES}

[1] Agile RF Transceivers and Front-Ends for Future Smart MultiStandard Communication Applications, ARTEMOS project, ENIAC-JU, call 3, 2010, Available: http://www.artemos.eu.

[2] P. Choi, et al., "An Experimental Coin-Sized Radio for Extremely Low-Power WPAN (IEEE 802.15.4) Application at $2.4 \mathrm{GHz}, "$ in IEEE Journal of Solid-State Circuits, vol. 38, n. 12, pp. 2258-2268, Dec. 2003.

[3] F. Alimenti, et al, "A New Contactless Assembly Method for Paper Substrate Antennas and UHF RFID Chips," IEEE MTT Trans., vol.59, no.3, pp.627-637, March 2011.

[4] J. Thaysen, K.B. Jakobsen, and J. Appel-Hansen, "A Logarithmic Spiral Antenna for 0.4 to $3.8 \mathrm{GHz} ., "$ in Applied Microwave \& Wireless, Vol. 13, No. 2, 2001, p. 32-45.

[5] L. Yang, A. Rida, R. Vyas, and M. M. Tentzeris, "RFID tag and RF Structures on a Paper Substrate Using Inkjet-Printing Technology" IEEE Transaction on Microwave Theory and Techniques, vol. 55, no. 12, pp. 2894-2901, Dec. 2007. 